\title{
Re-administration of gefitinib following diffuse interstitial lung disease in a patient with advanced lung adenocarcinoma: A case report and review of the literature
}

\author{
XUELI ZHANG, HUIQIAO LI, MIN ZHU and YUHUI ZHANG \\ Beijing Institute of Respiratory Medicine, Beijing Key Laboratory of Respiratory and Pulmonary Circulation Disorders, \\ Beijing Chao-Yang Hospital, Capital Medical University, Beijing 100020, P.R. China
}

Received June 18, 2014; Accepted February 17, 2015

DOI: $10.3892 / 01.2015 .3026$

\begin{abstract}
Interstitial lung disease (ILD) is a severe adverse effect of gefitinib treatment. Re-administration of gefitinib to patients suspected of suffering from gefitinib-induced ILD requires cautious consideration. In the majority of cases, gefitinib is not re-administered to such patients. The present study reports a case of advanced lung adenocarcinoma, where the patient developed gefitinib-induced ILD and gefitinib was re-administered at 3.5 months after discontinuation of gefitinib treatment. Initially, the patient achieved partial clinical remission, but developed diffuse ILD following gefitinib administration for 5 months. Following the onset of ILD, gefitinib was discontinued immediately and low-dose corticosteroids were administered at the early stages of ILD. Subsequent to recovery from the lung injury, gefitinib was re-administered along with $\mathrm{N}$-acetylcysteine. The patient presented with good lung adenocarcinoma control and did not experience a recurrence of ILD for $>16$ months. Thus, early discontinuation and gefitinib re-administration with $\mathrm{N}$-acetylcysteine may be a potential novel treatment strategy for gefitinib-induced ILD.
\end{abstract}

\section{Introduction}

Gefitinib,aninhibitorofepidermal growthfactorreceptor(EGFR) tyrosine kinase, is an effective and well-tolerated drug used for the treatment of advanced non-small cell lung cancer (NSCLC) $(1,2)$. However, gefitinib treatment may result in a number of severe adverse effects, including the development of interstitial lung disease (ILD) (3-7). Although certain studies have been conducted on the safety of re-administering gefitinib to patients who were suspected of suffering from gefitinib-induced ILD,

Correspondence to: Professor Yuhui Zhang, Beijing Institute of Respiratory Medicine, Beijing Key Laboratory of Respiratory and Pulmonary Circulation Disorders, Beijing Chao-Yang Hospital, Capital Medical University, 8 Gongtinan Road, Chaoyang, Beijing 100020, P.R. China

E-mail: zhangyhcy@163.com

Key words: interstitial lung disease, gefitinib, adenocarcinoma no precise management strategy has been established for such cases (8-11). The present study reports the case of a patient with lung adenocarcinoma, initially responding to gefitinib treatment. However, the patient developed gefitinib-induced ILD, which was treated with low-dose corticosteroids at the early stages of ILD, and then gefitinib was re-administered along with $\mathrm{N}$-acetylcysteine.

\section{Case report}

A 64 year-old female nonsmoker was diagnosed with lung adenocarcinoma $\left(\mathrm{T}_{4} \mathrm{~N}_{2} \mathrm{M}_{1 \mathrm{~b}}\right)$ with multiple pulmonary and bone metastases in August 2011 at the Beijing Chao-Yang Hospital (Beijing, China). The patient received four cycles of chemotherapy with gemcitabine (1400 mg, d1; $1600 \mathrm{mg}, \mathrm{d} 8$ ) plus cisplatin (40 mg, d2-d3; $30 \mathrm{mg}, \mathrm{d} 4$ ) as the first-line therapy (21 day cycles, 4 cycles). A brain MRI scan in November 2011 revealed multiple metastases in the patient, indicating disease progression.

In February 2012, after 2 months of whole-brain radiotherapy (dosage, 40Gy/20F) yielding a partial response, gefitinib was administered at a dose of $250 \mathrm{mg} /$ day as the second-line therapy. However, an EGFR mutation test was not performed as not enough lung cancer tissue was obtained. After 5 months (July 4, 2012), the tumor exhibited partial response; however, a high-resolution computerized tomography (HRCT) scan revealed the presence of diffuse ground glass opacities (GGOs) in the lungs (Fig. 1). Since no respiratory symptoms or evidence of infections or collagen vascular diseases were observed, the patient was diagnosed with gefitinib-induced ILD. Gefitinib administration was immediately discontinued, and prednisone was administered orally at a lower dose of $20 \mathrm{mg} /$ day, which was reduced by $5 \mathrm{mg} /$ week. After a further 2 weeks later (July 20, 2012), an HRCT scan revealed that the size of the GGOs reduced. After an additional 3.5 months (October 30, 2012), the GGOs disappeared; however, the primary cancer remained unchanged (Fig. 2). Since the patient refused further chemotherapy and a marked antitumor response to the gefitinib treatment was previously observed, gefitinib was re-administered at the same dose as before, along with $\mathrm{N}$-acetylcysteine (dosage, $600 \mathrm{mg}$ PO tid for 11 months). The patient 

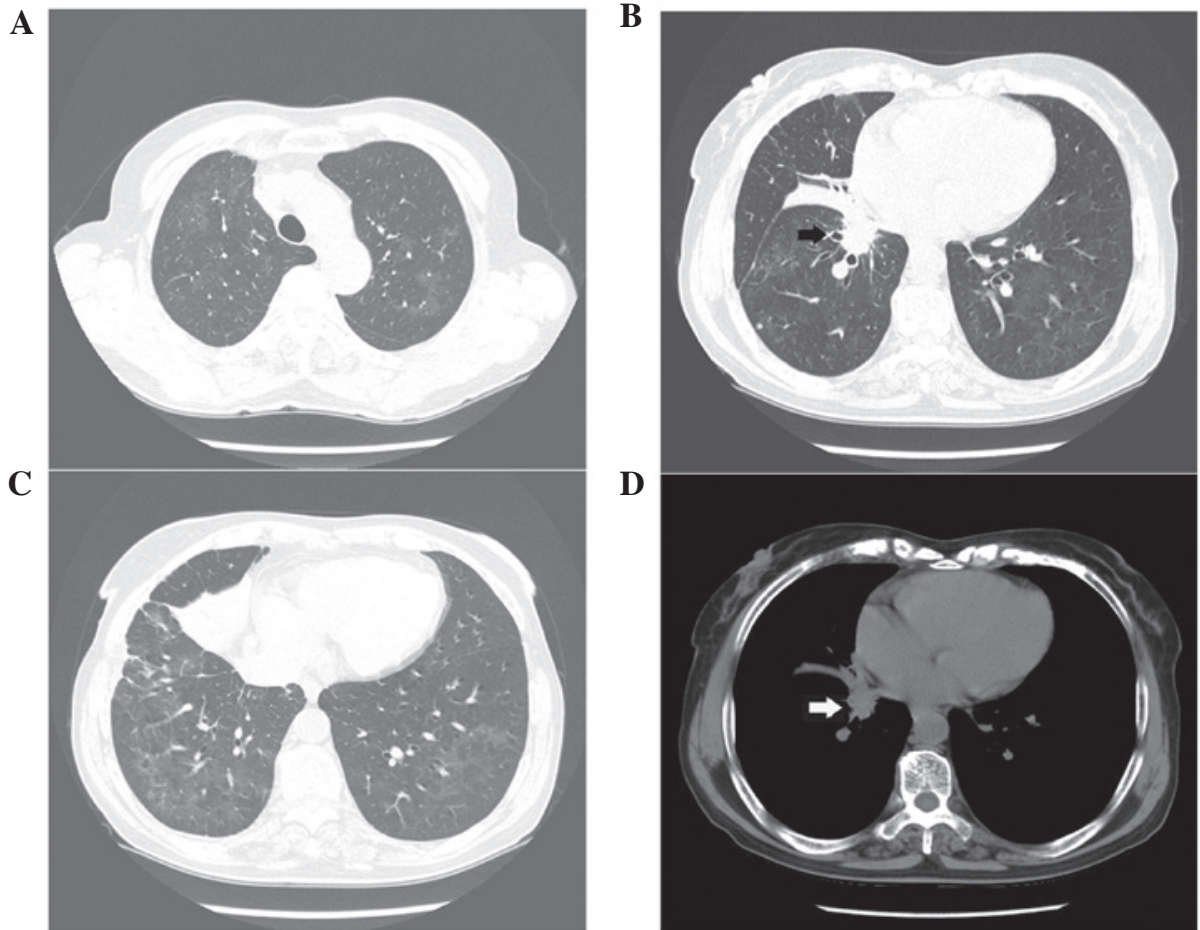

Figure 1. High-resolution CT scans showing (A-C) three different scanning planes of the lung. These revealed that gefitinib-induced interstitial lung disease was developed, presenting as ground glass opacities in the lungs 5 months after the initial gefitinib therapy, and (D) plain CT of primary cancer demonstrating partial regression (July 4,2012). The arrows show the primary cancer. CT, computerized tomography.
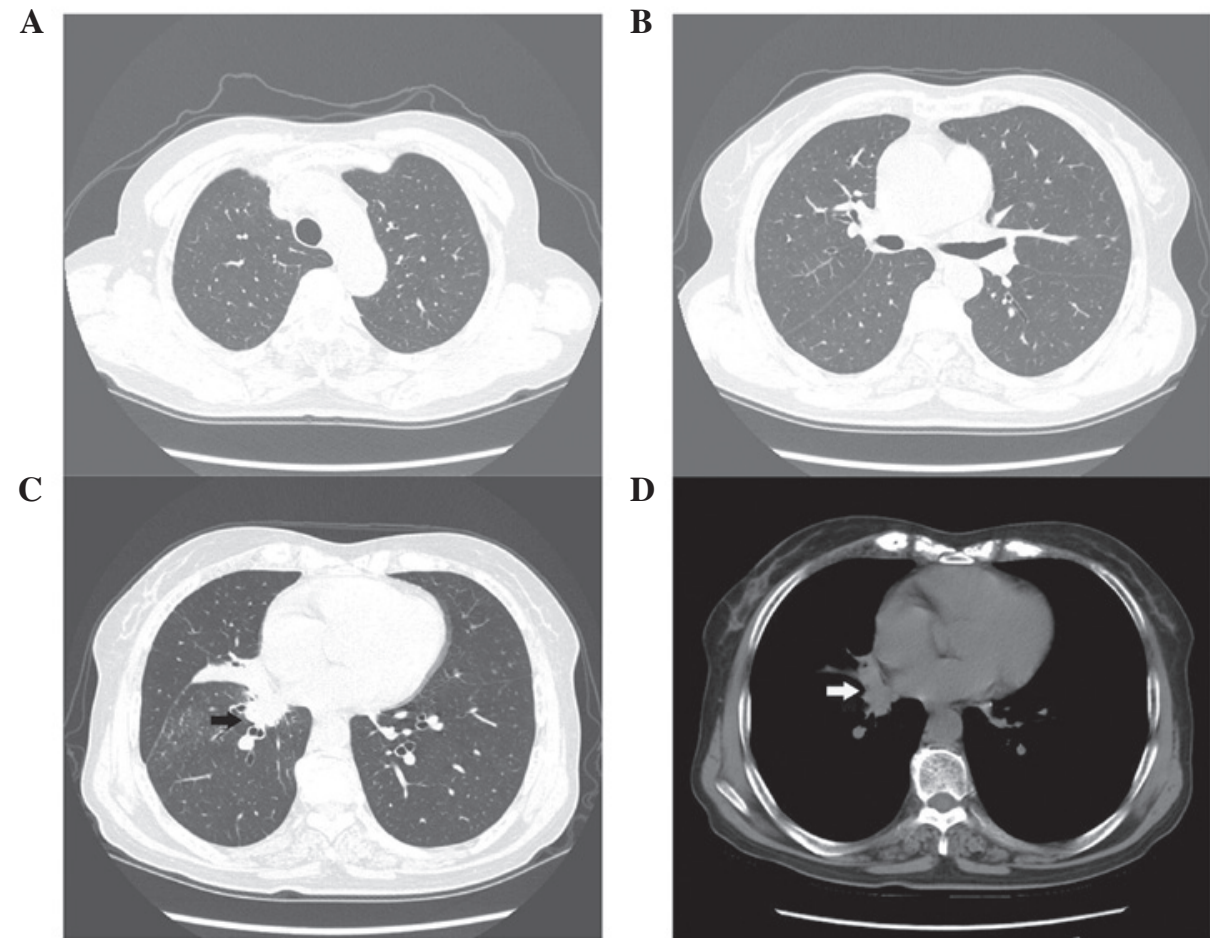

Figure 2. High-resolution CT scans showing (A-C) three different scanning planes of the lung. These revealed that the ground glass opacities disappeared 3.5 months after discontinuation of gefitinib. (D) Plain CT of the primary cancer demonstrated partial regression (October 30, 2012). The arrows show the primary cancer. CT, computerized tomography.

achieved 20 months of progression-free survival (PFS) and 30.5 months of overall survival (OS), until April 15, 2014. No GGOs were detected in HRCT images captured during the 16-month period prior to the patient succumbing to lung cancer-induced respiratory failure. The current study was approved by the ethics committees of Beijing Chao-Yang Hospital, Capital Medical University and written informed consent was provided by the patient's family. 


\section{Discussion}

Gefitinib-induced ILD has been reported to occur in $\sim 4.0 \%$ of patients treated with gefitinib in a study on the Japanese population (12). To date, no precise management strategy exists for gefitinib-induced ILD. Immediate withdrawal of gefitinib, administration of corticosteroids and oxygen therapy are currently recommended (13). Previous reported cases of gefitinib-induced ILD have typically been treated with a high-dose of corticosteroids (intravenous injection of $1 \mathrm{~g}$ /day methylprednisolone for three consecutive days, followed by 50 or $60 \mathrm{mg} /$ day oral prednisone, which is reduced by $10 \mathrm{mg} /$ week) or with the cessation of gefitinib administration, which result in ILD recovery or mortality $(9,11)$. Intravenous cyclophosphamide has also been reported as a valid treatment (10). In the present study, gefitinib was discontinued immediately following the onset of ILD, and a low-dose of corticosteroids were administered at an early stage.

Takamochi et al (9) reported the re-administration of gefitinib in a patient who developed gefitinib-induced ILD. A chest CT scan revealed a significant response following gefitinib re-administration at a $50 \%$ dose $(125 \mathrm{mg} /$ day $)$ for a month. However, Suzuki et al (8) reported a case of recurrent gefitinib-induced ILD following re-administration of gefitinib at a dose of $250 \mathrm{mg} /$ day in a patient with severe ILD.

In the present study, the initial gefitinib treatment was associated with a remarkable antitumor response, and no evidence of other adverse events induced by gefitinib was detected, with the exception of ILD. Thus, gefitinib was re-administered along with $\mathrm{N}$-acetylcysteine and the patient was under close observation. $\mathrm{N}$-acetylcysteine is a thiol-containing compound that contains sulfhydryl groups, which function as a glutathione precursor or as reduced glutathione, serving as direct scavengers of reactive oxygen species (14). $\mathrm{N}$-acetylcysteine is commonly used to treat patients with idiopathic pulmonary fibrosis (15-17). The patient of the present study exhibited an encouraging response to gefitinib treatment and achieved long PFS and OS periods. The clinical benefits observed in the present case suggest that the management of gefitinib-induced ILD requires further investigation. Early discontinuation and gefitinib re-administration along with $\mathrm{N}$-acetylcysteine may be a potential new treatment strategy for patients suffering from lung tumors and gefitinib-induced ILD.

\section{Acknowledgements}

This study was supported by a grant from the Wu Jieping Medical Foundation (grant no. 320.6750.12623) and by an educational grant from the Ministry of Education of China (grant no. 201347; 2014).

\section{References}

1. Fukuoka M, Yano S, Giaccone G, et al: Multi-institutional randomized phase II trial of gefitinib for previously treated patients with advanced non-small-cell lung cancer (The IDEAL 1 Trial) [corrected]. J Clin Oncol 21: 2237-2246, 2003.

2. Kris MG, Natale RB, Herbst RS, et al: Efficacy of gefitinib, an inhibitor of the epidermal growth factor receptor tyrosine kinase, in symptomatic patients with non-small cell lung cancer: a randomized trial. JAMA 290: 2149-2158, 2003.

3. Inoue A, Saijo Y, Maemondo M, et al: Severe acute interstitial pneumonia and gefitinib. Lancet 361: 137-139, 2003.

4. Okamoto I, Fujii K, Matsumoto M, et al: Diffuse alveolar damage after ZD1839 therapy in a patient with non-small cell lung cancer. Lung Cancer 40: 339-342, 2003.

5. Ricciardi S, Tomao S and de Marinis F: Toxicity of targeted therapy in non-small-cell lung cancer management. Clin Lung Cancer 10: 28-35, 2009.

6. Ando M, Okamoto I, Yamamoto N, et al: Predictive factors for interstitial lung disease, antitumor response and survival in non-small-cell lung cancer patients treated with gefitinib. J Clin Oncol 24: 2549-2556, 2006

7. Luo C, Lv M, Li Y, et al: Gefitinib-induced interstitial pneumonia: A case report and review of the literature. Exp Ther Med 7: 855-859, 2014.

8. Suzuki M, Asahina H, Konishi J, Yamazaki K and Nishimura M: Recurrent gefitinib-induced interstitial lung disease. Intern Med 47: 533-536, 2008.

9. Takamochi K, Suzuki K, Bashar AH, et al: Readministration of gefitinib in a responder after treatment discontinuation due to gefinitib-related interstitial lung disease: a case report. J Med Case Rep 1: 138, 2007.

10. Goto Y, Hojo M, Takeda Y, Kobayashi N and Kudo K: Gefitinib-induced interstitial lung disease-addition of intravenous cyclophosphamide to corticosteroids is a valuable treatment option: A case report. Med Oncol 27: 753-755, 2010.

11. Seto T, Seki N, Uematsu K, et al: Gefitinib-induced lung injury successfully treated with high-dose corticosteroids. Respirology 11: 113-116, 2006.

12. Kudoh S, Kato H, Nishiwaki Y, et al; Japan Thoracic Radiology Group: Interstitial lung disease in Japanese patients with lung cancer: a cohort and nested case-control study. Am J Respir Crit Care Med 177: 1348-1357, 2008.

13. Kitajima H, Takahashi H, Harada K, et al: Gefitinib-induced interstitial lung disease showing improvement after cessation: disassociation of serummarkers. Respirology 11: 217-220, 2006.

14. Gillissen A: Anti-inflammatory efficacy of N-acetylcysteine and therapeutic usefulness. Pneumologie 65: 549-557, 2011 (In German).

15. Albera C, Ferrero C, Rindone E, Zanotto S and Rizza E: Where do we stand with IPF treatment? Respir Res 14 (Suppl 1): S7, 2013.

16. Behr J and Richeldi L: Recommendations on treatment for IPF. Respir Res 14 (Suppl 1): S6, 2013.

17. Idiopathic Pulmonary Fibrosis Clinical Research Network; Raghu G, Anstrom KJ, King TE Jr, Lasky JA and Martinez FJ: Prednisone, azathioprine and N-acetylcysteine for pulmonary fibrosis. N Engl J Med 366: 1968-1977, 2012. 